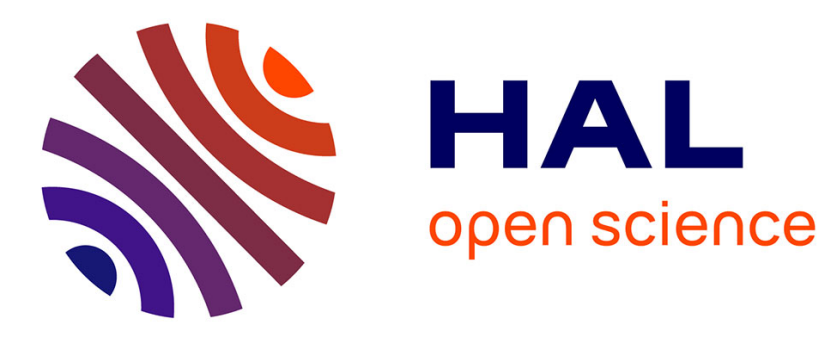

\title{
Toward Intuitive 3D User Interfaces for Climbing, Flying and Stacking
}

\author{
Antonin Bernardin, Guillaume Cortes, Rebecca Fribourg, Tiffany Luong, \\ Florian Nouviale, Hakim Si-Mohammed
}

\section{- To cite this version:}

Antonin Bernardin, Guillaume Cortes, Rebecca Fribourg, Tiffany Luong, Florian Nouviale, et al.. Toward Intuitive 3D User Interfaces for Climbing, Flying and Stacking. VR 2018 - IEEE Conference on Virtual Reality and 3D User Interfaces, Mar 2018, Reutlingen, Germany. pp.860-861, 10.1109/VR.2018.8446047 . hal-01949784

\section{HAL Id: hal-01949784 \\ https://hal.inria.fr/hal-01949784}

Submitted on 8 Jan 2019

HAL is a multi-disciplinary open access archive for the deposit and dissemination of scientific research documents, whether they are published or not. The documents may come from teaching and research institutions in France or abroad, or from public or private research centers.
L'archive ouverte pluridisciplinaire HAL, est destinée au dépôt et à la diffusion de documents scientifiques de niveau recherche, publiés ou non, émanant des établissements d'enseignement et de recherche français ou étrangers, des laboratoires publics ou privés. 


\section{Toward Intuitive 3D User Interfaces for Climbing, Flying and Stacking}

\author{
Antonin Bernardin* \\ Univ Rennes, Inria, CNRS, \\ IRISA \\ Guillaume Cortes ${ }^{\dagger}$ \\ Realyz, Univ Rennes, Inria, \\ CNRS, IRISA \\ Florian Nouviale \\ Univ Rennes, Inria, CNRS, \\ IRISA
}

\author{
Rebecca Fribourg \\ Univ Rennes, Inria, CNRS, \\ IRISA \\ Hakim Si-Mohammed" \\ Univ Rennes, Inria, CNRS, \\ IRISA
}

\author{
Tiffany Luong $\$$ \\ Univ Rennes, Inria, CNRS, \\ IRISA
}

\begin{abstract}
In this paper we propose 3D user interfaces (3DUI) that are adapted to specific Virtual Reality (VR) tasks: climbing a ladder using a puppet metaphor, piloting a drone thanks to a $3 \mathrm{D}$ virtual compass and stacking $3 \mathrm{D}$ objects with physics-based manipulation and time control. These metaphors have been designed to provide the user with an intuitive, playful and efficient way to perform each task.
\end{abstract}

\section{Index Terms: Virtual Reality-3D User Interfaces-}

\section{INTRODUCTION}

Today's advancements in Virtual Reality (VR) led the community to question the basis of human interaction with a Virtual Environment (VE). Some of the most simple and inner tasks that humans regularly do such as walking, cannot be easily translated into VEs and require extensive work to be performed as naturally as in the real world. On the other hand, VEs offer the possibility to consider tasks which are complex or even impossible to perform in the real world (like flying). In front of these challenges, designing intuitive, realistic and simple interaction techniques is one of the key objectives of VR community. In this paper we propose 3D user interfaces for performing three tasks: climbing a virtual ladder with a puppet metaphor, piloting a drone in first person view thanks to a $3 \mathrm{D}$ virtual compass and stacking virtual objects with physics-based manipulation and time control.

\section{Ladder Climbing}

The ladder climbing task consists in climbing a ladder respecting some constraints to prevent a fall. For this reason, we decided to design a safe and efficient solution in terms of physical efforts, without requiring costly equipment. Our approach uses what we call the puppet metaphor, that does not require tracking of the feet but only requires tracking of both hands. Some user feedback was also implemented in order to help the user during the climbing and prevent a fall.

\subsection{The Puppet Metaphor}

We propose a puppet metaphor that allows to control the feet and the hands through two Vive controllers. For this purpose, the users have the possibility to control their limbs as they would control a puppet. Nevertheless, they are not immersed in an out-of-body situation, but remain in a first-person point of view. The position of their head is tracked using the Vive HMD and 3D models of the controllers map the real controllers position and orientation in the VE. We differentiate these controllers in the VE with the actual virtual hands and feet. Such virtual hands and feet are moved by the user over the ladder and are considered as being the puppet

\footnotetext{
*e-mail: antonin.bernardin@inria.fr

$\dagger$ e-mail: g.cortes@realyz.fr

¥e-mail: rebecca.fribourg@inria.fr

$\S$ e-mail: tiffany.luong@inria.fr

Ie-mail: florian.nouviale@inria.fr

"e-mail: hakim.si-mohammed@inria.fr
}
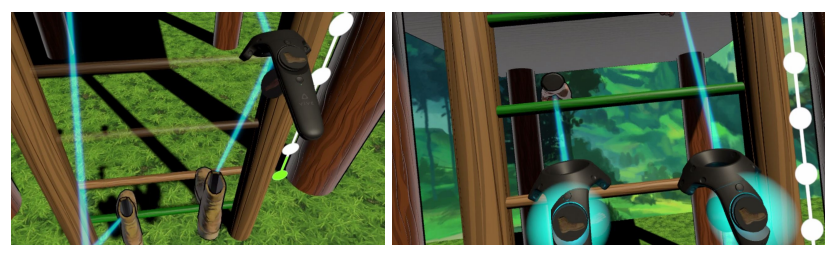

Figure 1: First person point of view of the ladder climbing. The user is observing either his/her feet (left) or his/her hands (right).

(Figure 1). Virtual strings link the controllers to the feet and hands. The users can choose to move their left (respectively right) foot or their left (respectively right) hand by pressing specific buttons on the controllers. In our solution are observed two kinds of user representation. There is the 3D controllers models that represent the real controllers and thus follow users hand movements. This display is the direct visual feedback of users movements, giving them the possibility to interact with their virtual puppet. This virtual puppet also represents the user. It was decided not to use a whole body representation of the user. Indeed, it was considered that eventually by only representing the limbs, the ladder visibility would be enhanced. Also, it is demonstrated in [1] that users may feel a strong sense of agency towards their action in the VE even without any body representation, which reinforces our choice of only partly representing users' body.

\subsection{Feedback Mechanisms}

As previously mentioned, when climbing the ladder the users are confronted with the risk of falling if a certain amount of constraints are not respected. To avoid that and preserve a good climbing experience, some visual and tactile feedback were implemented. During the climbing the users firstly press a specific button to move a selected limb, and then have to put it in collision with a rung of the ladder. When there is collision between a limb and a rung, the rung is highlight in blue, indicating that the user can release the button pressed so the limb can be fixed to the highlighted rung. The rungs with at least one limb fixed to them are highlighted in green. Also, as a constraint consists in making the users fall if their hands and feet are too close or too distant, we advice the users to either raise their hands or their feet to avoid the fall (dynamic arrows, highlight around specific buttons). In addition, some vibrations are provided through the controllers when a limb collides with a rung.

\section{First-PERSON DRONe Flying}

The drone task consists in piloting a drone and make it go through several rings that form a predefined circuit. For piloting the drone, which can be considered as a 3D navigation task, we chose to implement a 3D virtual compass metaphor that provides the drone with a direction to go towards. In [3] two interaction techniques for a way-finding task are compared, either by steering through the VE regardless of the gaze direction, or by coupling the gaze direction to the direction of travel. In this paper, we extend this approach to a 4 degrees of freedom navigation, using a 3D compass navigation 

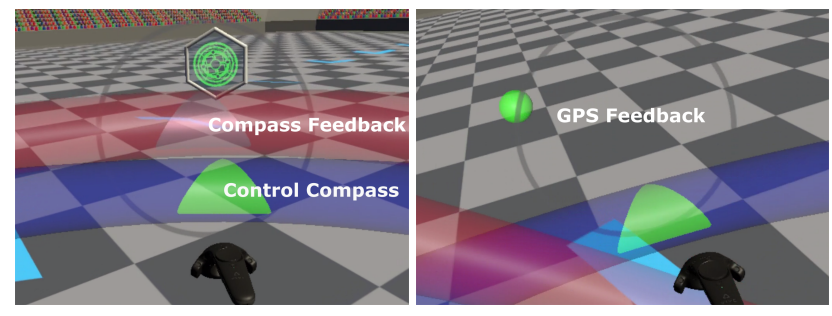

Figure 2: The 3D User Interface used to control the drone. The compass metaphor (left) and the GPS feedback (right) are depicted.

mechanism to control the drone. We also chose to implement some user feedback to provide information about the current state of the task.

\subsection{D Virtual Compass Metaphor}

The $3 \mathrm{D}$ virtual compass metaphor is used as a $3 \mathrm{D}$ navigation technique to allow the user to pilot the drone. The idea is to provide the user with a way to indicate the direction he wants the drone to go. This compass points in the direction defined by the vector going from the user's head to the user's hand. In other words, the user's position represents the center of the compass, and his/her arm represents the needle (Figure 2). In order to control the direction of the drone, the user points his/her arm in the desired direction. The resulted direction is represented on the blue compass with a green arrow. Finally the user can increase the power of the engines to control the speed by pressing the trigger. This interaction metaphor presents the advantage of abstracting the drone commands from yaw, pitch and roll angular velocities, to a direct and more intuitive pointing control system. Thus the user only has to handle the speed and direction of the drone.

\subsection{Feedback Mechanisms}

A first visual feedback is used to provide information about the drone physical orientation and direction, in the form of a transparent compass concentric to the control compass (Figure 2 left). Since the drone does not change its direction instantaneously (due to its physical model), this compass feedback indicates the current direction the drone is moving towards to. Then, in order to enable the users to locate themselves in the VE, a GPS like feedback is provided at the center of the users' field of view by means of a transparent gray circle (Figure 2 right). Whenever the user loses sight of the next ring to pass, a transparent green sphere is displayed on the border of the circle to indicate the direction of the missed ring. Finally, a vibro-tactile feedback is provided to the user thanks to the inner vibrations of the controller. This vibration indicates the power level of the engines. The stronger the vibration, the faster the propellers are spinning.

\section{ObJect Stacking}

The stacking task consists in stacking several physical objects so that a given height can be reached during ten seconds. Physicsbased hand interaction [2] is complex to handle in VR due to the offset between the almost completely-free motion of the user and the physical constraints of the VE. We propose a solution for physicsbased object manipulation as well as a time control feature to help the user perform the task with as much precision and control as possible.

\subsection{Physics-based Manipulation}

The first step of physics-based manipulation is to be able to select an object. Such selection is possible when a controller intersects an object. The object turns green if it can be manipulated. In that case the user can press the trigger of the corresponding controller to grab
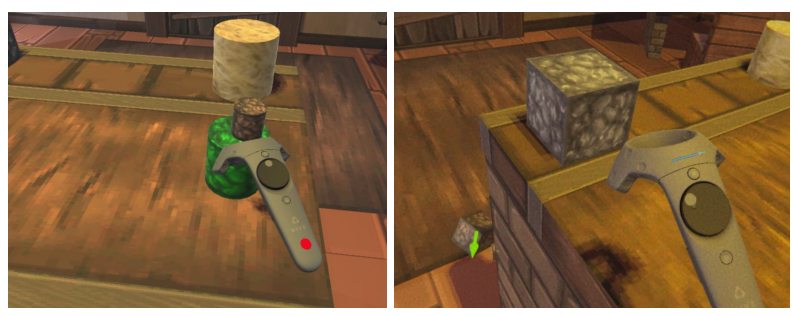

Figure 3: Physical object manipulation (left) with frame recording as indicated by the red round on the controller and time control (right), the green arrow indicates the object velocity, the pad can be rotated to navigate in the time-line as shown by the blue slider on the controller

it. The manipulation ends when the trigger is released which is close to a natural grab interaction.

An object is manipulated using its velocity and angular velocity, which avoids a strong coupling between the controller and the object it moves and allows this object to keep physical interactions with the rest of the scene. The manipulated object can thus collide with the other elements in the scene (Figure 3 left). At each instant, the position and rotation of the manipulated object will try to reach the ones of the controller with some spring-like effect, giving the user a sense of the physical constraints he performs to the manipulated object. When the trigger is released, one last velocity is applied. This velocity is proportional to the controller velocity at the time of the release, allowing the user to throw objects in a natural way.

\subsection{Time Control}

While objects are moving, the time control system records the position, orientation, velocity and angular velocity of each object. To go back in time, the user has to touch the pad of the right controller. A screen effect (with grain and color desaturation) will be enabled so that the user knows in which state the system is. A virtual wheel, shown on the controller can be rolled clockwise or counter-clockwise to navigate in the recorded past states of the scene. A slider bar also indicates the position of the current timestep in the recorded timeline. The user can continue to move freely when controlling time and green arrows indicate the recorded velocities of the objects at the given timestep (Figure 3 right). When the user pulls any of the triggers, the time control system will stop and the simulation will resume at the state set by the user. Every object will hence continue its movement corresponding to the recorded velocities and angular velocities recorded for that timestep.

\section{CONCLUSION}

In this paper, we propose intuitive and creative solutions to perform dedicated tasks in virtual environments such as climbing a ladder, piloting a drone and stacking objects. A puppet metaphor was implemented to climb the ladder. The drone is piloted using a 3D virtual compass metaphor. Finally the stacking is performed with physics-based manipulation and time control. Those interaction techniques pave the way to further studies aiming to improve 3D user interfaces.

\section{REFERENCES}

[1] F. Argelaguet, L. Hoyet, M. Trico, and A. Lecuyer. The role of interaction in virtual embodiment: Effects of the virtual hand representation. In IEEE VR, pp. 3-10, 2016.

[2] D. Bowman, E. Kruijff, J. J. LaViola Jr, and I. P. Poupyrev. 3D User Interfaces: Theory and Practice, Chapter 5.4.3. Direct Manipulation: Virtual Hand Techniques. Addison-Wesley, 2004.

[3] C. Christou, A. Tzanavari, K. Herakleous, and C. Poullis. Navigation in virtual reality: Comparison of gaze-directed and pointing motion control. In MELECON, pp. 1-6, 2016. 